\title{
Metallothionein-IIA Promotes Initial Neurite Elongation and Postinjury Reactive Neurite Growth and Facilitates Healing after Focal Cortical Brain Injury
}

\author{
Roger S. Chung, James C. Vickers, Meng Inn Chuah, and Adrian K. West \\ NeuroRepair Group, School of Medicine, University of Tasmania, Hobart, Tasmania 7001, Australia
}

\begin{abstract}
Metallothioneins (MTs) are small, cysteine-rich, metal binding proteins. Their function has often been considered as stress-related proteins capable of protecting cells from heavy metal toxicity and oxidative free radicals. However, recent interest has focused on the brain-specific MT-III isoform, which has neurite-inhibitory properties. To investigate the effect of another MT isoform, human MT-IIA, on neurite growth, we used rat cortical neuron cultures. MT-IIA promoted a significant increase in the rate of initial neurite elongation of individually plated neurons. We also investigated the effect of MT-IIA on the neuronal response to axonal transection in vitro. MT-IIA promoted reactive axonal growth after injury, and, by $18 \mathrm{hr}$ after transection, MT-IIA had promoted axonal growth across the injury tract.

Exogenous application of MT-IIA after cortical brain injury promoted wound healing, as observed by a significant decrease in cellular degradation at $4 \mathrm{~d}$ after injury. Furthermore, MT-IIA-treated rats exhibited numerous SMI-312-immunoreactive axonal processes within the injury tract. This was in contrast to vehicle-treated animals, in which few axonal sprouts were observed. By $7 \mathrm{~d}$ after injury, MT-IIA treatment resulted in a total closing over of the injury tract by microglia, astrocytes, and reactive axonal processes. However, although some reactive axonal processes were observed within the injury tract of vehicle-treated rats, the tract itself was almost never entirely enclosed. These results are discussed in relation to a possible physiological role of metallothioneins in the brain, as well as in a therapeutic context.
\end{abstract}

Key words: metallothionein; reactive sprouting; neurite sprouting; neurite growth; cortical brain injury; wound healing

\section{Introduction}

The metallothionein (MT) family of proteins consists of cysteinerich $(25-30 \%)$, low molecular weight $(6-7 \mathrm{kDa})$, heavy metal binding proteins (Hamer, 1986; Kagi and Schaffer, 1988; Vasak and Hasler, 2000). There are four isoforms: I and II (found in most tissues), III (found primarily in the brain), and IV (found in squamous epithelial tissue) (Palmiter et al., 1992; Quaife et al., 1994; Blaauwgeers et al., 1996). The MT-I and MT-II isoforms are remarkably similar (both structurally and in both spatial and temporal expression profiles), so much so that they are often treated as a single isoform (denoted MT-I/-II).

Remarkably, the precise function of the MTs remains elusive, despite years of study (Palmiter, 1998). It is known that their heavy metal binding properties allow them to sequester heavy metals and protect against metal toxicity (in particular cadmium), as well as regulating the availability of metal ions, particularly $\mathrm{Zn}$ (II) and $\mathrm{Cu}$ (I), to various enzymes and transcription factors (Kelly et al., 1996; Aschner et al., 1997). They also have free radical scavenging properties, which allow them to protect cells from cytotoxicity induced by reactive oxygen species (Sato and Bremner, 1993). Perhaps most intriguing is the possible roles

\footnotetext{
Received Sept. 9, 2002; revised Feb. 3, 2003; accepted Feb. 3, 2003.

This work was funded by the Motor Accident and Insurance Board of Tasmania and the Australian National Health and Medical Research Council. Our thanks go to Bedrich Eckhardt for production of the human MT-IIA expression vector. We also thank Julie Harris, Jyoti Chuckowree, and Graeme McCormack for their assistance throughout this work.

Correspondence should be addressed to Roger S. Chung, NeuroRepair Group, School of Medicine, University of Tasmania, P.0. Box 252-58, Hobart, Tasmania 7001, Australia. rschung@postoffice.utas.edu.au. Copyright $\odot 2003$ Society for Neuroscience $\quad 0270-6474 / 03 / 233336-07 \$ 15.00 / 0$
}

that MTs may have in relation to neurodegenerative diseases, such as Alzheimer's disease (AD) (Uchida et al., 1991; Adlard et al., 1998). Although it is the brain-specific MT-III isoform that has been investigated most in this context, there is recent evidence to suggest that the MT-I/-II isoforms may also have important roles in the CNS and in neurodegenerative diseases. Indeed, we recently found MT-I/-II to be specifically upregulated in the preclinical stages of AD (Adlard et al., 1998). Furthermore, recent work from Penkowa et al. (1999a,b, 2002) suggests that MT-I/-II are intimately involved in wound healing after brain injury and in experimental autoimmune encephalomyelitis, an animal model of multiple sclerosis (Penkowa and Hidalgo, 2000).

MT-III has been observed to inhibit neuronal survival (Uchida et al., 1991; Erickson et al., 1994) and neurite sprouting and growth (Uchida et al., 1991, 2002; Chung et al., 2002b) in culture. Interestingly, other MT isoforms (namely MT-I and -II) exhibit antiapoptotic properties (Abdel-Mageed and Agrawal, 1998; Penkowa et al., 1999a). To investigate the effect of these isoforms on neurite sprouting and growth, we used embryonic rat cortical neuron cultures. We found that human MT-IIA promoted neurite elongation during initial neurite development and growth. After axonal transection of cultured neurons (21 d in vitro), MT-IIA promoted regenerative axonal growth. Furthermore, exogenous application of MT-IIA after cortical brain injury promoted wound healing, as assessed by cellular degradation and reactive axonal sprouting.

\section{Materials and Methods}

Chemicals and antibodies. Trypsin, trypsin inhibitor, L-glutamine, gentamicin, poly-L-lysine, and rabbit anti-mouse MAP-2 (microtubuleassociated protein-2) primary antibody were supplied from Sigma (St. 
Louis, MO). Neurobasal medium and B-27 supplement were purchased from Invitrogen (Auckland, New Zealand). Anti-tau, MT-I/-II, and GFAP antibodies were from Dako (Glostrup, Denmark). Anti- $\beta$ IIItubulin antibody was from Promega (Madison, WI). Anti-neurofilament M (NF-M) antibody was purchased from Serotec (Oxford, UK). SMI312 was obtained from Sternberger Monoclonals (Lutherville, MD). Anti-ferritin antibody was from ICN Biomedicals (Costa Mesa, CA). Alexafluor secondary antibodies were from Molecular Probes (Eugene, OR). Horse anti-mouse rat adsorbed IgG conjugated to fluoroscein was obtained from Vector Laboratories (Burlingame, CA).

Construction of the recombinant human MT-IIA and MT-III expression vectors. The recombinant human MT-IIA and MT-III vectors were prepared as reported previously (Erickson et al., 1994), using reverse transcription-PCR subclones in the bacterial expression vector pET-3d (Novagen, Madison, WI). The structure of the construct was confirmed by DNA sequencing. Recombinant MT protein expression was induced in the presence of $\mathrm{ZnSO}_{4}$ and was purified as described previously. After anion-exchange chromatography, purified MT was then acidified with dilute $\mathrm{HCl}$ to $\mathrm{pH} 2.0$, and bound $\mathrm{Zn}$ (II) ions were removed by applying the sample to an ultrafiltration column. The apo-MT was stored in aliquots at $-80^{\circ} \mathrm{C}$ until required. When required, the apo-MT was reconstituted with $\mathrm{Zn}$ (II) by the addition of $7.5 \mathrm{~mol}$ equivalents followed by neutralization of the sample to $\mathrm{pH} 8.0$ with $20 \mathrm{~mm}$ Tris-HCl. The sample was desalted by ultrafiltration.

Single-cell neuron cultures. Primary neuron cultures were prepared as described previously (Chung et al., 2002a). Briefly, cortical tissue was removed from embryonic day 18 (sperm positive day is embryonic day 1) Hooded Wistar rat embryos and incubated in sterile $10 \mathrm{~mm}$ HEPES buffer $\left(37^{\circ} \mathrm{C}\right)$. This was followed by trypsin digestion $(0.25 \%)$, followed by addition of trypsin inhibitor (40 benzoyl-L-tyrosine ethyl ester units per milligram of protein). After three washes with HEPES buffer, the cell suspension was then triturated carefully using a $1 \mathrm{ml}$ pipette. The cell suspension was filtered through gauze of pore size $60 \mu \mathrm{m}$, and cells were counted by trypan blue exclusion. Cells were then plated onto glass coverslips $\left(132 \mathrm{~mm}^{2}\right)$ precoated overnight with poly-L-lysine $(0.01 \%)$, at a cell density of $1 \times 10^{5}$ cells per well, with $150 \mu \mathrm{g} / \mathrm{ml}$ adult rat brain extract added. Brain extract was prepared as described previously (Chung et al., 2002a). Unless stated otherwise, MT-IIA (0.01, 0.1, 1, and $5 \mu \mathrm{g} / \mathrm{ml}$ ) was added at this time. Cultures were maintained at $37^{\circ} \mathrm{C}$ in humidified air containing $5 \% \mathrm{CO}_{2}$ for $3 \mathrm{~d}$ after MT addition. The culture medium consisted of Neurobasal medium, supplemented with $0.1 \%$ (final concentration) B-27 supplement, $0.1 \mathrm{~mm}$ (final concentration) L-glutamine, and $200 \mathrm{U} / \mathrm{ml}$ gentamicin.

Three days after initial plating, cells were fixed with $4 \%$ paraformaldehyde for $20 \mathrm{~min}$. Fluorescent double immunocytochemistry using an anti-MAP-2 monoclonal primary antibody (1:1000 dilution) and nuclear yellow staining was used to determine the neuron culture purity. Analysis was performed by capturing 10 digital images from six different coverslips, from three individual cultures (different embryos). Ninetyeight percent of cells were identified as MAP-2-immunoreactive neurons (results not shown).

Neuron cluster cultures. Previously, we observed that cortical neurons cultured at high density form clusters, with thick fasciculated axonal bundles forming between them (Dickson et al., 2000). Furthermore, reactive axonal sprouting is observed after transection of the axonal bundles. Neurons were obtained as described above, except that they were plated directly from the unfiltered cell suspension onto glass coverslips $\left(254 \mathrm{~mm}^{2}\right)$ at a cell density of $4.5 \times 10^{5}$ cells per well and were incubated overnight at $37^{\circ} \mathrm{C}, 5 \% \mathrm{CO}_{2}$. These coverslips had been precoated overnight with poly-L-lysine $(0.01 \%)$. The next day, the culture medium was replaced with fresh medium, and the neurons were maintained for $21 \mathrm{~d}$ (initial plating is day 0 ), with the medium replaced with fresh medium every $4 \mathrm{~d}$. The medium used was as described previously for single-cell neuron cultures. The formation of neurite bundles between neuronal clusters became apparent by $7 \mathrm{~d}$ after plating.

Transection of axonal bundles. Transection of axonal bundles was as described previously (Dickson et al., 2000). Briefly, at $20 \mathrm{~d}$ after initial plating, coverslips were moved to individual Petri dishes $\left(\sim 2900 \mathrm{~mm}^{2}\right)$ with $150 \mu \mathrm{g} / \mathrm{ml}$ rat brain extract added. The following day, axonal bun- dles were transected on an inverted microscope (Fluovent; Leitz, Wetzlar, Germany) using a fine goniotomy knife. Immediately after transection, either $5 \mu \mathrm{g} / \mathrm{ml}$ MT-IIA or an equivalent volume of $0.1 \%$ PBS was added, and the cultures were maintained for 4,12 , or $18 \mathrm{hr}$. In some cases, parallel experiments were performed, and $1 \mu \mathrm{g} / \mathrm{ml}$ MT-III was applied to cultures immediately after transection.

Fluorescent immunocytochemistry of cultured neurons. At the appropriate time, cells were fixed with $4 \%$ paraformaldehyde for $20 \mathrm{~min}$. Coverslips were then incubated overnight with either anti-NF-M $(1: 1000)$ or both anti-tau $(1 ; 5000)$ and anti- $\beta$ III-tubulin $(1: 10,000)$ primary antibodies diluted in $0.1 \%$ PBS and $0.03 \%$ Triton X-100. Anti-NF-M reacts with the medium neurofilament subunit (Karlsson et al., 1987). Anti-tau binds with the microtubule-associated tau protein (Goedart et al., 1988). Anti- $\beta$ III-tubulin binds with the major building block of microtubules and is neuron specific (Lee et al., 1990). Coverslips were then incubated with two secondary antibodies (horse anti-mouse IgG conjugated to Alexafluor 488 and goat anti-rabbit IgG conjugated to Alexafluor 594; 1:1000 dilution), applied in $0.1 \%$ PBS. Coverslips were mounted onto slides using Permafluor mounting medium (Immunotech, Marseilles, France).

Calculations and analysis of cultured neurons. For analysis, 10 digital phase contrast images were captured systematically, two from each quadrant and two from the central area, for each time point $(2,4,24,48$, and $72 \mathrm{hr}$ after plating), at a magnification of $400 \times$ (Olympus BL-51; Olympus Optical, Tokyo, Japan). Each group, unless otherwise stated, consisted of three coverslips. The total number of cells counted per coverslip ranged from 300 to 500 cells.

Neurites were defined as any process of at least $20 \mu \mathrm{m}$ in length extending from the cell body. The percentage of neurite-bearing neurons (or more accurately the percentage of neurons with one or more neurites) was calculated by dividing the average number of neurite-bearing neurons per field by the average total number of neurons per field. Of the neurite-bearing neurons, the number of neurites per cell was calculated by dividing the average number of neurites per field by the average number of neurite-bearing neurons per field. ANOVA analysis of results was performed using SigmaStat (Jandel Scientific, San Rafael, CA).

Neurite length measurements were made using NIH Image, with at least 300 neurites being measured per treatment group. To determine the rate of neurite elongation between time points, the average length of neurites at the earlier time point was subtracted from the average length of neurites at the later time point.

For postaxonal transection analysis in the neuron cluster cultures, five digital images of different injury sites of each coverslip were taken (Olympus BX-60). Each group (representing the time points 4, 12, and $18 \mathrm{hr}$ after transection) consisted of at least three coverslips. Experiments were repeated in triplicate (three different neuronal cultures). All measurements were made using NIH Image. $t$ test analysis of results was performed using SigmaStat.

Anti-NF-M immunolabeled digital images were used to measure the area of retraction between transected neuritic stumps. At least 15 injury sites were measured per group of three coverslips. Anti-tau/ $\beta$ III-tubulinimmunolabeled digital images were used for measurements of neurite length. Neurites were measured from the proximal end (defined by the punctately distributed $\beta$ III-tubulin) to the distal tip. At least 150 neurites were measured per group of three coverslips.

Rat focal cortical injuries. All procedures involving animals were approved by the Animal Experimentation Ethics Committee of the University of Tasmania and are consistent with the Australian Code of Practice for the Care and Use of Animals for Scientific Purposes. Rat focal cortical injuries were made as reported previously (King et al., 1997). Briefly, 250 gm male rats were anesthetized with $0.1 \mathrm{ml} / 100 \mathrm{gm} \mathrm{Nembutal}$ (sodium pentobarbitol, intraperitoneal), the head was shaved, and the rat was then immobilized in a Stoelting (Kiel, WI) stereotaxic frame. An incision down the midline of the skull was made, and a hole was carefully drilled through the skull above the Par 1 region of the somatosensory cortex using a Dremel surgery drill. A Hamilton syringe with a 25 gauge beveled needle was aligned with the hole and then lowered $1.5 \mathrm{~mm}$ into the brain. After $10 \mathrm{~min}$, the syringe was slowly removed, and the hole was filled with Gelfoam, pretreated with either $20 \mu \mathrm{l}$ of PBS or $20 \mu \mathrm{l}$ of MT-IIA $(5 \mu \mathrm{g} / \mathrm{ml}$ 
concentration). Antiseptic powder was applied, and then the skin was pulled back over the skull and held together by two staples.

A total of 16 rats were treated with MT-IIA (in four different experiments) and maintained for $4 \mathrm{~d}$ after injury. The same number of control animals (treated with PBS) were also used. An additional 12 rats received MT-IIA (in four different experiments) for $7 \mathrm{~d}$ postinjury studies, and a similar number of animals received vehicle (PBS).

Fluorescent immunohistochemistry of rat brain sections. At the appropriate time, rats were reanesthetized and transcardially perfused with $4 \%$ paraformaldehyde. Brains were removed and postfixed overnight in $4 \%$ paraformaldehyde at $4^{\circ} \mathrm{C}$. They were then embedded in $5 \%$ agar (in $0.1 \% \mathrm{PBS}$ ) and sectioned by vibratome at a thickness of $50 \mu \mathrm{m}$. Sections were then used in fluorescent immunohistochemistry as described below or stored in PBS (containing $0.01 \%$ sodium azide) at $4^{\circ} \mathrm{C}$.

For immunohistochemistry, the sections were incubated overnight with a combination of two monoclonal primary antibodies: SMI-312/ anti-ferritin $(1: 2000 ; 1: 10,000)$ or anti-MT-I/-II/anti-GFAP (1:500; 1:2000). Anti-ferritin was used as a marker of activated microglial cells (King et al., 2001). SMI-312 labels the phosphorylated forms of neurofilaments and is an axonal marker (Ulfig et al., 1998). The antibodies were diluted in $0.1 \%$ PBS and $0.03 \%$ Triton X-100. Sections were then incubated with two secondary antibodies (goat anti-rabbit IgG conjugated to Alexafluor 488, 1:250 dilution; and horse anti-mouse rat adsorbed IgG conjugated to fluoroscein, 1:250 dilution) applied in 0.1\% PBS. Brain sections were mounted using Permafluor mounting medium. Specimens were viewed on the BX-60 (Olympus Optical) fluorescence microscope.

The volume of tissue exhibiting ferritin immunoreactivity was used as a measure of inflammatory response. The volume of the inflammatory response to injury was determined as the sum of the volume of ferritin immunoreactivity in the epicentrical tissue section and the next two consecutive sections in either direction. The percentage of tissue degradation was determined by subtracting the area of empty space from the total area of ferritin immunoreactivity within a brain section. For both of these analyses, nine animals (over three different experiments) were used.

\section{Results}

\section{MT-IIA has no effect on initial neurite formation}

To investigate the effect of MT-IIA on initial neurite formation, neurons were plated immediately in the presence of MT-IIA and grown for $3 \mathrm{~d}$. Analysis of both the neurites per neuron (Fig. 1A) and the percentage of neurite-bearing neurons (Fig. $1 B$ ) indicated that, at both protein concentrations tested $(0.1$ and $1 \mu \mathrm{g} /$ $\mathrm{ml}$ ), MT-IIA had no significant effect on initial neurite formation ( $p>0.01$; ANOVA). The vehicle- and MT-IIA-treated cultures resulted in $\sim 80 \%$ of neurons forming neurites, and each cell formed an average of two neurites by $3 \mathrm{~d}$.

\section{MT-IIA significantly increases the rate of neurite elongation} To investigate whether MT-IIA has an effect on the rate of growth of developing neurites, measurements of neurite length were made from cultures up to $3 \mathrm{~d}$ after MT-IIA treatment. In the vehicle-treated group, there was an initial retraction by the neurites (between 0 and $2 \mathrm{hr}$ ), most likely in response to the medium change at this time (Fig. $2 \mathrm{~A}$ ). From $2 \mathrm{hr}$ onward, the rate of neurite elongation steadily increased.

In the presence of MT-IIA (at $1 \mu \mathrm{g} / \mathrm{ml}$ ), however, the initial neurite retraction between 0 and $2 \mathrm{hr}$ was absent, with the neurites elongating during this time period (Fig. $2 \mathrm{~A}$ ). Furthermore, from $4 \mathrm{hr}$ onward, there was a significant $(p<0.01$; Student's $t$ test) dose-dependant increase in the rate of neurite elongation compared with vehicle treated (Fig. $2 A$ ), indicating that MT-IIA promotes neurite elongation.

Vehicle-treated neurons projected neurites that had extended an average length of $138.7 \pm 7.5 \mu \mathrm{m}$ by $3 \mathrm{~d}$. In the presence of MT-IIA, however, the average neurite length was significantly
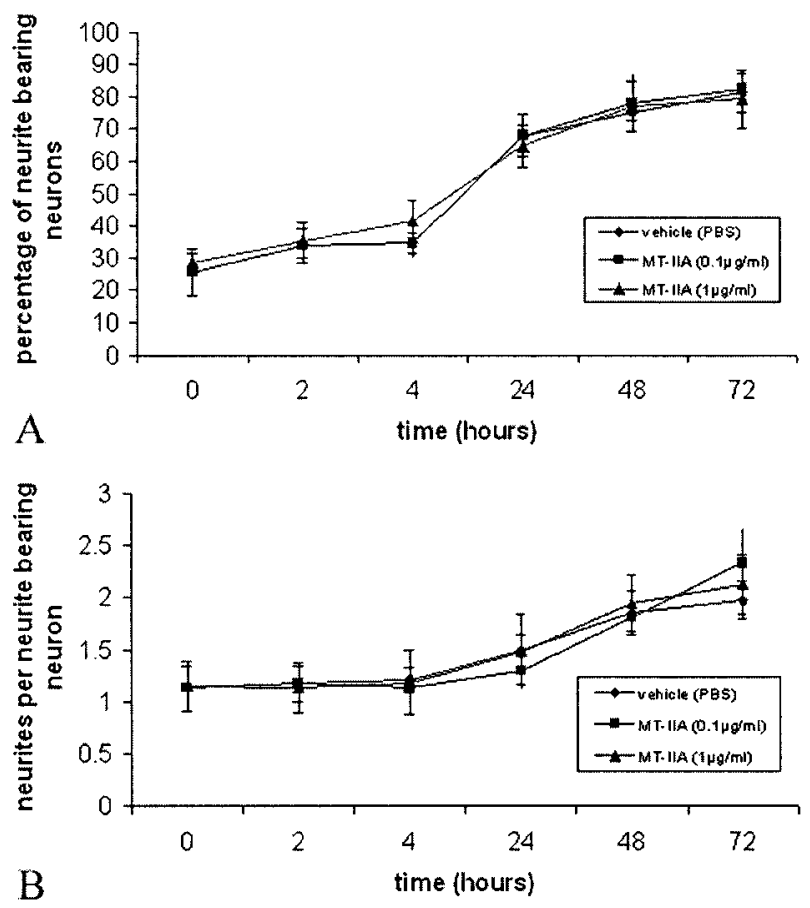

Figure 1. Human MT-IIA had no effect on initial neurite outgrowth over $3 d$, as assessed by both the percentage of neurite-bearing neurons $(A)$ or number of neurites per neuron $(B)(p>$ 0.01 ; ANOVA). For all graphs, error bars represent SE values.
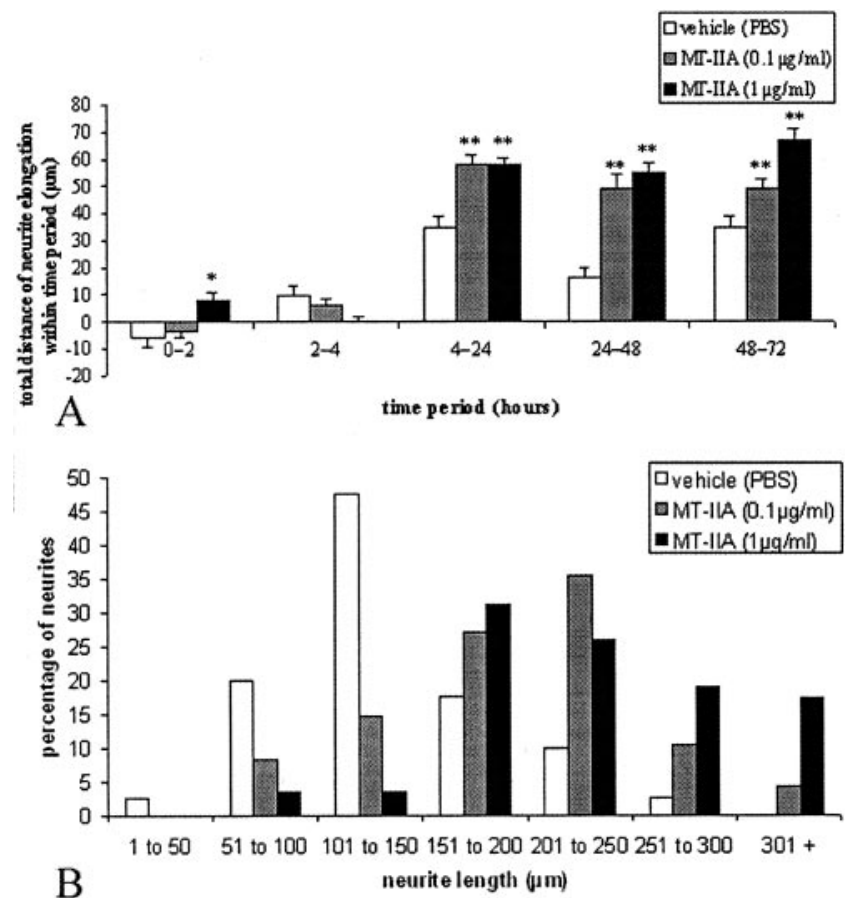

Figure 2. MT-IIA dose dependently promoted neurite elongation up to $72 \mathrm{hr}$ in vitro $(A)$. The total neurite length at $3 \mathrm{~d}$ after MT-IIA treatment $(B)$ also indicates that MT-IIA significantly promoted neurite growth. ${ }^{*} p<0.05 ;{ }^{* *} p<0.01$; Student's $t$ test. For all graphs, error bars represent SE values.

increased to $203.9 \pm 10.3$ and $234.3 \pm 9.7 \mu \mathrm{m}(0.1$ and $1 \mu \mathrm{g} / \mathrm{ml}$ MT-IIA respectively; $p<0.01$; Student's $t$ test). Analysis of the distribution of neurite lengths clearly indicates that MT-IIA promotes neurite growth (Fig. $2 B$ ). 

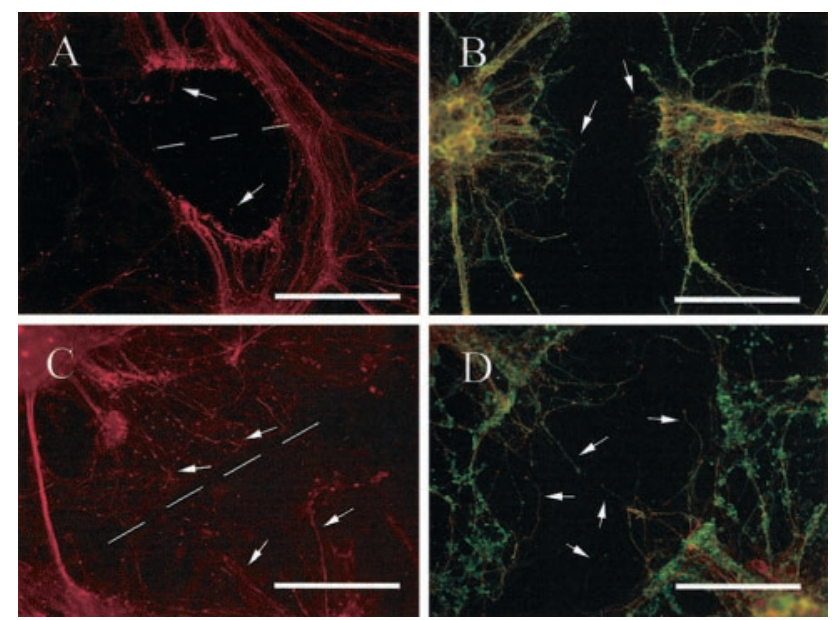

Figure 3. At $12 \mathrm{hr}$ after injury, there was a marked retraction by transected neurites from the lesion site (indicated by the broken line) of up to $100 \mu \mathrm{m}$. Although there were very few NF-M-immunoreactive processes (red) extending into the area of retraction (indicated by arrows) in vehicle-treated neurons $(A)$, there were many in MT-IIA-treated neurons ( $C$ ). Tau (red) and $\beta$ III-tubulin (green) immunocytochemical analysis also indicated very few processes extended into the area of retraction (indicated by arrows; $B$ ). In the presence of MT-IIA, these processes were significantly longer (D). Scale bars: $A, B, 100 \mu \mathrm{m} ; C, 200 \mu \mathrm{m} ; D, 50 \mu \mathrm{m}$.

Effect of MT-IIA on the neuronal response to injury: neuronal cluster model of reactive axonal sprouting

In analogy to the response of neurons in vivo after traumatic injury, the process of reactive axonal sprouting is observed after axonal transection to established neurons in culture (Dickson et al., 2000) and is interpreted as part of the subsequent neuronal regenerative process. To investigate the effect that MT-IIA has on this neuronal response in culture, MT-IIA was applied to cultures immediately after axonal transections in cluster cultures.

\section{MT-IIA promotes the growth of reactive neurite sprouts after injury}

At $4 \mathrm{hr}$ after injury, there were clear cytoskeletal changes present in response to injury. These changes took the form of NF-Mimmunoreactive, ring-like structures (Dickson et al., 2000). MTIIA had no effect on the development of these structures, in either quantity or morphology (results not shown). At this time, there were few, very short $(<10 \mu \mathrm{m})$ tau/ $\beta$ III-tubulin processes extending from the transected neurite stumps, even in the presence of MT-IIA (results not shown). As described previously (Dickson et al., 2000), there was a marked retraction by the transected neurites, often resulting in a concave surface at the cut edge.

By $12 \mathrm{hr}$ after, the injured neurites had retracted further from the point of injury. This retraction was highlighted by NF-Mimmunoreactive rings present away from the point of injury, resulting in an area of retraction between the two transected neurite stumps ranging from 90 to $220 \mu \mathrm{m}$ in length (mean length, $146.8 \pm 10.4 \mu \mathrm{m})$. There were very few NF-M-immunoreactive processes extending into the area of retraction (Fig. $3 A$ ). Tau/ $\beta$ III-tubulin-immunoreactive processes were also identified extending into the area of retraction (Fig. $3 B$ ). $\beta$ III-Tubulin immunoreactivity at this and later time points was found to often be punctately distributed in preexisting neurites, accumulating at the ends of the transected neurite stumps. This $\beta$ III-tubulin accumulation was used as another indicator of the neuritic retraction after injury.

In the presence of MT-IIA, neuritic retraction (indicated by NF-M-immunoreactive rings) was also present and was not sig-
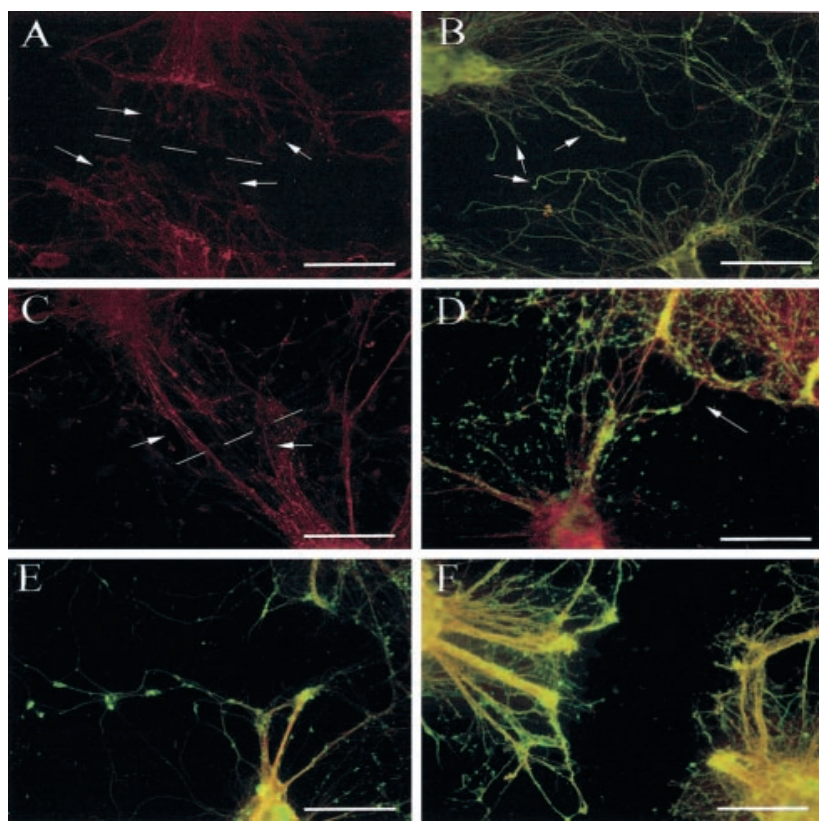

Figure 4. NF-M immunocytochemical analysis of neurite transections $18 \mathrm{hr}$ after injury, both vehicle treated $(A)$ and MT-IIA treated $(C)$. NF-M-immunoreactive processes (red) extended from the neurite stumps (indicated by arrows) and grew toward the central lesion site (indicated by the broken line). MT-IIA promoted growth of NF-M-immunoreactive processes (indicated by arrows) across the central lesion site (C). Tau (red) and $\beta$ III-tubulin (green) immunocytochemical analysis also indicated a number of processes that extended into the area of retraction (indicated by arrows) in vehicle-treated neurons, but again these processes were not observed crossing the transection site $(B)$. MT-IIA promoted the growth of processes across the transection site (indicated by arrows) and to the opposite stump of the transected neurite bundle $(D)$. In contrast, MT-III significantly inhibited reactive neurite sprouting $(E, F)$. Scale bars: $A, C-F, 100$ $\mu \mathrm{m} ; B, 50 \mu \mathrm{m}$.

nificantly different from the control (results not shown). Furthermore, there were a number of NF-M-immunoreactive processes extending into the area of retraction (Fig. $3 C$ ). There were also a number of tau/ $\beta$ III-tubulin-immunoreactive processes extending across the area of retraction (Fig. $3 D$ ). Neurite measurements indicate that the MT-IIA-treated tau/ $\beta$ III-tubulin processes (mean neurite length, $70.1 \pm 3.4 \mu \mathrm{m}$ ) were significantly longer than vehicle-treated processes (mean neurite length, $41.5 \pm 1.7 \mu \mathrm{m})$ at this time point $(p<0.01$; Student's $t$ test $)$. Furthermore, there appeared to be more reactive processes per $100 \mu \mathrm{m}$ of cut site length after MT-IIA treatment $(20.8 \pm 0.8)$ than in vehicle-treated cultures $(15.8 \pm 1.2)$, although this was not a statistically significant difference $(p=0.07$; Student's $t$ test).

By $18 \mathrm{hr}$ after transection, there were a number of NF-Mimmunoreactive processes extending through the area of retraction. However, no reactive processes were observed crossing to the opposing side of the transection site (Fig. $4 A$ ). Tau/ $\beta$ IIItubulin-immunoreactive processes also extended through the area of retraction but also did not cross the entire length of the transection site (Fig. 4B).

In the presence of MT-IIA, however, a number of NF-Mimmunoreactive processes were identified crossing the entire transection site (Fig. 4C). A small number of sprouts had bulblike neurofilament accumulations within them. Tau/ $\beta$ IIItubulin-immunoreactive processes were also identified crossing the transection site and continued to the opposing transected neurite stump (Fig. 4D). This was in contrast to the effect of the other major neural isoform of MT, MT-III, which significantly 

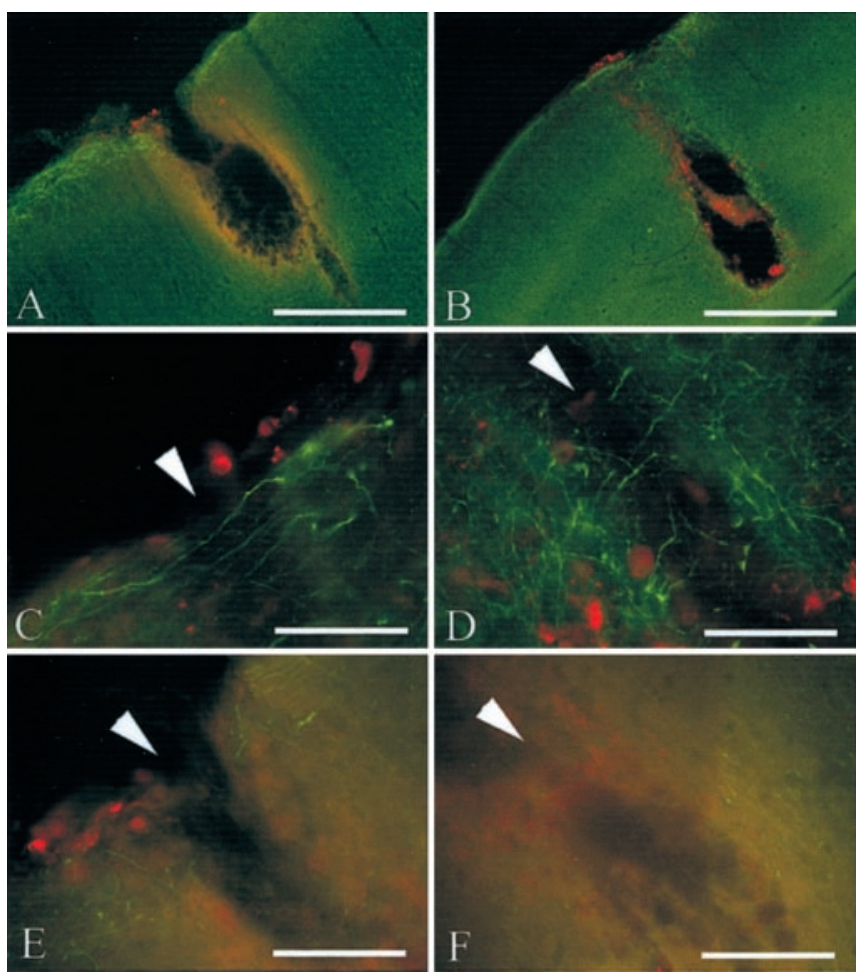

Figure 5. Brain sections underwent immunohistochemistry against SMI-312 (green) and ferritin (red) at $4 \mathrm{~d}$ after injury. Needle stick injury resulted in a large injury tract and microglial migration into and surrounding the injury site $(A)$. MT-IIA treatment promoted the formation of a tissue bridge enclosing the lesion site from the pial surface down, forming a teardrop-like invagination ( $B$ ). MT-IIA promoted axonal sprouting into the lesion site at both the pial layer $(C)$ and deeper cortical layers $(D)$. In contrast, very few axonal sprouts were visualized in control rats, at the pial level $(E)$ or deeper cortical layers $(F)$. Arrowheads indicate the injury tract. Scale bars: $A, B, 100 \mu \mathrm{m} ; C-E, 25 \mu \mathrm{m}$.

inhibited reactive neurite sprouting (Fig. $4 E, F$ ), indicating that neither metallothioneins, per se, nor protein-bound zinc are responsible for the observed enhancement of sprouting.

\section{Exogenous MT-IIA promotes wound healing and reactive axonal growth after cortical brain injury}

Recombinant human MT-IIA was exogenously applied into the lesion site (in Gelfoam placed directly above the lesion) after cortical needle stick injury. At $4 \mathrm{~d}$ after injury, the area of the injury site was clearly demarcated by ferritin immunoreactivity (Fig. 5A). The volume of inflammatory response (defined by ferritin immunoreactivity) was not significantly affected by MT-IIA application ( $p=0.096$; Student's $t$ test), although the volume in all MT-IIA-treated animals (average volume, $2035 \mathrm{~mm}^{3}$ ) was smaller than vehicle-treated rats (average volume, $2772 \mathrm{~mm}^{3}$ ). From the pial surface down, MT-IIA treatment resulted in the formation of a cellular bridge enclosing the injury site, resulting in a teardrop-like injury site (Fig. 5B). The percentage of tissue degradation within the area of inflammation was significantly greater within vehicle-treated animals ( $54 \pm 4$ vs $12 \pm 11 \%$; $p<$ $0.01 ; t$ test).

MT-IIA promoted the growth of a number of SMI-312immunoreactive axonal processes into the injury site, at all cortical levels injured, as well as at the pial surface (Fig. $5 C, D$ ). This was in marked contrast to vehicle-treated rats, in which very few SMI-312-immunoreactive processes were visualized entering the lesion site (Fig. 5E,F). As reported previously, these SMI-312immunoreactive reactive axonal sprouts exhibited a higher de-
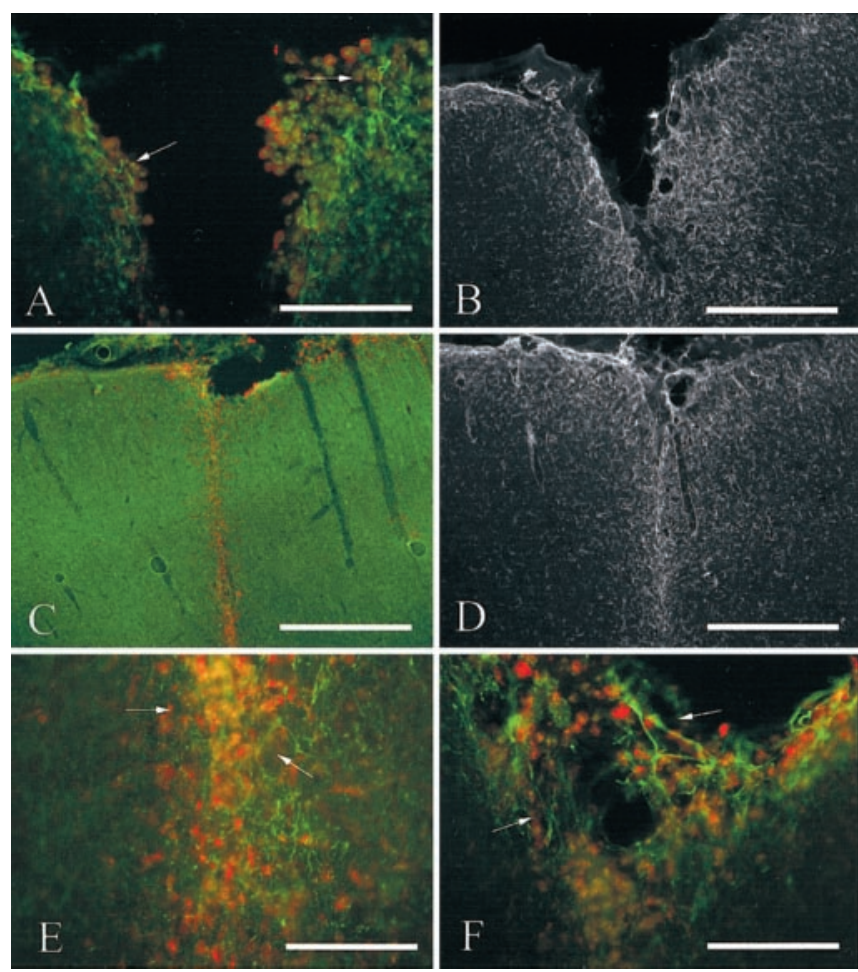

Figure 6. Immunohistochemical staining against SMI-312 (green), ferritin (red), and GFAP (black and white images) of brain sections at $7 \mathrm{~d}$ after injury. In vehicle-treated rats, the injury tract was smaller compared with $4 \mathrm{~d}$ after injury, although it had not completely closed over. $\mathrm{A}$ degree of reactive sprouting was evident (arrows) in all animals at this time point ( $A$ ). Reactive processes exhibited greater $\mathrm{SMI}-312$ reactivity than background neuritic processes. Reactive astrocytes also aligned along the borders of the injury tract $(B)$. In MT-IIA-treated rats, the entire injury tract had closed over and was demarcated only by a fine line of ferritin immunoreactivity $(C)$. Reactive astrocytes also enclosed the injury tract and were found at lower density in adjacent uninjured tissue (D). In MT-IIA-treated animals, numerous reactive axonal processes were observed (arrows) within the injury tract, at both deeper cortical levels $(E)$ and the pial level $(F)$. Scale bars: $A, 50 \mu \mathrm{m} ; B-D, 100 \mu \mathrm{m} ; E, F=25 \mu \mathrm{m}$.

gree of labeling for neurofilaments than adjacent, uninjured processes (King et al., 2001).

By $7 \mathrm{~d}$ after injury in vehicle-treated rats, the injury tract was significantly smaller than at $4 \mathrm{~d}$ after injury, although a cavity was often evident (Fig. 6A). Reactive sprouting was observed in all vehicle-treated animals at this time point (Fig. 6A). Reactive astroglial migration resulted in a thick line along the borders of the injury tract, and reactive astrocyte density gradually diminished away from the injury site (Fig. $6 \mathrm{~B}$ ). In MT-IIA-treated rats, the entire injury tract was enclosed. Microglial inflammation was decreased and was found as a thin line of ferritin-immunoreactive cells demarcating the injury site (Fig. 6C). Astrocytes also formed a thin line marking the injury tract. The density of reactive astrocytes decreased further from the injury site (Fig. 6D). Numerous SMI-312immunoreactive processes were observed within the injury tract, although these processes often did not exhibit a higher degree of labeling than neighboring, uninjured processes (Fig. 6 E). At the pial surface however, long reactive axonal processes were observed entering the injury site, which exhibited increased labeling compared with adjacent, uninjured processes (Fig. $6 F$ ).

\section{Discussion}

Whereas the neuroactive properties of MT-III have been well documented, there is little known about other MT isoforms. This is surprising given the high structural similarity between the var- 
ious MT isoforms. We report that, after axonal transection in culture, MT-IIA promotes regenerative neurite growth. In addition, exogenous application of MT-IIA after cortical brain injury resulted in promotion of the wound healing response, as observed by decreased cellular degradation, and a significant temporal progression in the presence of reactive axonal processes.

Although we found that MT-IIA does not affect initial neurite outgrowth of individually plated neurons, it does significantly promote the rate of neurite elongation ( $p<0.01$; ANOVA). Furthermore, by $12 \mathrm{hr}$ after axonal transection, MT-IIA-treated neurites had extended almost twice as far as vehicle-treated neurites (mean neurite length, $70.1 \pm 3.4$ and $41.5 \pm 1.7 \mu \mathrm{m}$, respectively), and, by $18 \mathrm{hr}$ after injury, MT-IIA had promoted neurite growth to the opposing transected neurite stump (vehicle-treated neurites were not found to extend across the entire injury area by this time). It has been suggested that the cytoskeletal events driving the reactive sprouting response recapitulate those that occur during initial neurite growth (Hoffman and Cleveland, 1988; Lee and Cleveland, 1996). It is plausible to postulate then that our observations of the effect of MT-IIA on postinjury reactive axonal growth may be attributable to the ability of MT-IIA to promote neurite elongation, which we observed during initial neurite growth. The fact that there were also significantly more regenerating sprouts present after injury, both in culture and in vivo, may indicate that MT-IIA is also promoting survival of transected axons and their associated cell bodies, such that they are able to make a regenerative sprouting response. This would be in accordance with the fact that MT-IIA did not promote initial neurite formation in developing neurons. As an additional indication of this, MT-I/-II has been reported previously to promote neuronal survival after various experimental models of neuronal injury (Campagne et al., 1999; Penkowa et al., 2002).

Exogenous application of MT-IIA after cortical brain injury significantly promoted wound healing, as observed by a significant decrease in cellular degradation, and an increase in reactive axonal growth into the injury tract. These results are similar to that reported in MT-I overexpressing mice (Campagne et al., 1999; Giralt et al., 2002), MT-I/-II knock-out mice (Penkowa et al., 1999a; Trendelenburg et al., 2002), or mice receiving Zn-MT-II injections after focal freeze brain injury or 6-aminonicotinamide treatment (Giralt et al., 2002; Penkowa et al., 2002), in which MT significantly improved wound healing. However, we further identified the presence of a number of SMI312-immunoreactive axonal processes within the injury tract at $4 \mathrm{~d}$ after that were not present in vehicle-treated animals. By $7 \mathrm{~d}$ after injury, a number of axonal sprouts were observed within the injury tract, which was also significantly smaller than vehicletreated animals. On the basis of our work and that of others (as discussed above), we propose that MT is able to enhance wound healing by two distinct mechanisms. Penkowa et al. (1999a,b, 2000) have reported significant evidence to suggest that MT-I/-II is essential for a number of postinjury recovery processes, such as astrocytic activation and migration, as well as decreasing inflammatory responses (such as microglial activation and proinflammatory cytokine expression). However, our work suggests that MT-I/-II may also additionally enhance wound healing by acting directly on neurons, by promoting regenerative axonal growth into the injury tract. Both of these mechanisms appear to be generic to the MT-I/-II isoforms, because we observed similar results with a commercial MT-I/-II source (results not shown; rabbit mixed Zn-MT-I/-II; Sigma).

Interestingly, the expression of MT-I/-II is rapidly and significantly upregulated after spinal cord injury (up to eightfold in- crease in expression) (Carmel et al., 2001), and in a number of different forms of human and experimental brain injury (for review, see Hidalgo et al., 2001). This poses the question of whether native, upregulated MT-I/-II are exerting the extracellular, neuroactive properties suggested by our work, after injury. MT-I/-II levels in the human cortex have been estimated at $\sim 40 \mu \mathrm{g} / \mathrm{gm}$ brain tissue (Erickson et al., 1994), and, given that necrosis or physical damage could cause extensive lysis of cells, it is possible that local extracellular concentrations of MT-I/-II could reach the levels that we and others have observed in the neuroactive properties of MTs in culture (i.e., in the vicinity of $1 \mu \mathrm{g} / \mathrm{ml}$ ). It must be noted, however, that, physiologically, MTs are considered solely intracellular proteins (Palmiter et al., 1992), and hence their functions are most often considered within an intracellular context. Recently, Uchida et al. (2002) demonstrated that cultured astrocytes actively release MT-III into medium (as lactate dehydrogenase was not released into the medium). Although it is not known whether MT-I/-II are actively released, there are numerous reports of the detection of MT-I/-II in the extracellular environment in vivo (Garvey, 1984; Bremner et al., 1987; Hidalgo et al., 1988) and in culture (Trayhurn et al., 2000). Further elucidation of extracellular MT-I/-II release mechanisms by astrocytes will improve our understanding of the role that these proteins play after wound healing and CNS disorders.

It is intriguing that the structurally related MT isoform MTIII exhibits opposing neuroactive properties to those we report here for MT-IIA. That is, MT-III possesses neurotoxic activity (Erickson et al., 1994) and also inhibits initial neurite formation and postinjury neurite sprouting in similar culture assays to those reported here (Chung et al., 2002b). Human MT-IIA and MT-III share a $70 \%$ sequence homology and also exhibit similar metal binding properties. Indeed, the 20 conserved cysteine residues, which characterize the mammalian MTs, are conserved across both isoforms. As an indication of this, Sewell et al. (1995) found that, by changing the $\mathrm{C}_{(6)}$ PCP motif of MT-III to the $\mathrm{C}_{(6)} \mathrm{SCT}$ motif found in MT-I/-II isoforms, the neurotoxicity of MT-III was abolished. Furthermore, engineering of the $\mathrm{T}_{(5)}$ CPCP motif into MT-I resulted in neurotoxic activity (Romero-Isart et al., 2002). The opposing neuroactive properties of these remarkably similar MT isoforms, combined with their specific spatial and temporal expression patterns both developmentally and after injury (for review, see Hidalgo et al., 2001), suggests that these neuroactive properties are specialized, true physiological functions of these proteins. Furthermore, the opposing action of $\mathrm{Zn}$ MT-III compared with Zn-MT-IIA that we observed both in culture and in vivo (Chung et al., 2002b; our unpublished results) strongly suggests that the neuroactive effects of MT-IIA are not attributable to the zinc moiety of the protein. As an additional indication of this, equivalent amounts of $\mathrm{Zn}$ did not exhibit similar effects to MT-IIA in culture (results not shown).

In summary, the present study demonstrates that exogenous application of the MT-I/-II isoform, human Zn-MT-IIA, after focal cortical injury, enhances wound healing and dramatically promotes reactive axonal growth, indicating a potential physiological role for MTs within the brain. Furthermore, it is an exciting possibility that the ability of MT-IIA to reduce inflammatory microglial response and promote reactive axonal growth may be of use as a therapeutic agent after neuronal injury. As an indication of this, Penkowa and Hidalgo (2001) demonstrated recently that intraperitoneal application of rat Zn-MT-II can promote recovery in experimental autoimmune encephalomyelitis, an animal model of multiple sclerosis. 


\section{References}

Abdel-Mageed AB, Agrawal KC (1998) Activation of nuclear factor kappaB: potential role in metallothionein-mediated mitogenic response. Cancer Res 58:2335-2338.

Adlard PA, West AK, Vickers JC (1998) Increased density of metallothionein I/II-immunopositive cortical glial cells in the early stages of Alzheimer's disease. Neurobiol Dis 5:349-356.

Aschner M, Cherian MG, Klaassen CD, Palmiter RD, Erickson JC, Bush AI (1997) Metallothioneins in brain - the role in physiology and pathology. Toxicol Appl Pharmacol 142:229-242.

Blaauwgeers HG, Anwar Chand M, van den Berg FM, Vianney de Jong JM, Troost D (1996) Expression of different metallothionein messenger ribonucleic acids in motor cortex, spinal cord and liver from patients with amyotrophic lateral sclerosis. J Neurol Sci 142:39-44.

Bremner L, Mehra RK, Sato M (1987) Metallothionein in blood, bile and urine. Toxicol 108:129-140.

Campagne MV, Thibodeaux H, van Bruggen N, Cairns B, Gerlai R, Palmer JT, Williams SP, Lowe DG (1999) Evidence for a protective role of metallothionein-1 in focal cerebral ischemia. Proc Natl Acad Sci USA 96:12870-12875.

Carmel JB, Galante A, Soteropoulos P, Tolias P, Recce M, Young W, Hart RP (2001) Gene expression profiling of acute spinal cord injury reveals spreading inflammatory signals and neuron loss. Physiol Genomics $7: 201-213$.

Chung RS, Holloway AF, Eckhardt BL, Harris JA, Vickers JC, Chuah MI, West AK (2002a) Sheep have an unusual variant of the brain specific metallothionein, MT-III. Biochem J 365:323-328.

Chung RS, Vickers JC, Chuah MI, Eckhardt BL, West AK (2002b) Metallothionein-III inhibits initial neurite formation in developing neurons as well as post-injury, regenerative neurite sprouting. Exp Neurol $178: 1-12$.

Dickson TC, Adlard PA, Vickers JC (2000) Sequence of cellular changes following localized axotomy to cortical neurons in glia-free culture. J Neurotrauma 17:1095-1103.

Erickson JC, Sewell AK, Jensen LT, Winge DR, Palmiter RD (1994) Enhanced neurotrophic activity in Alzheimer's disease cortex is not associated with down-regulation of metallothionein-III (GIF). Brain Res 649:297-304.

Garvey JS (1984) Metallothionein: structure/antigenicity and detection/ quantitation in normal physiological fluids. Environ Health Prosp 54:117-127.

Giralt M, Penkowa M, Lago N, Molinero A, Hidalgo J (2002) Metallothionein$1+2$ protect the CNS after a focal brain injury. Exp Neurol 173:114-128.

Goedart M, Wischik CM, Crowther RA, Walker JE, Klug A (1988) Cloning and sequencing of the CDNA encoding a core protein of the paired helical filament of Alzheimer's disease: identification as the microtubuleassociated protein tau. Proc Natl Acad Sci USA 85:4051-4055.

Hamer DH (1986) Metallothionein. Annu Rev Biochem 55:913-951.

Hidalgo J, Giralt M, Garvey JS, Armario A (1988) Physiological role of glucocorticoids on rat serum and liver metallothionein basal and stress conditions. Am J Physiol 254:E71-E78.

Hidalgo J, Aschner M, Zatta P, Vasak M (2001) Roles of the metallothionein family of proteins in the central nervous system. Brain Res Bull 55:133-145.

Hoffman PN, Cleveland DW (1988) Neurofilament and tubulin expression recapitulates the developmental program during axonal regeneration: induction of a specific beta-tubulin isotype. Proc Natl Acad Sci USA 85:4530-4533.

Kagi JH, Schaffer A (1988) Biochemistry of Metallothionein. Biochemistry 27:8509-8515.

Karlsson JE, Rosengren LE, Haglid KG (1987) A rapid HPLC method to separate the triplet proteins of neurofilament. J Neurochem 49:1375-1378.

Kelly EJ, Quaife CJ, Froelick GJ, Palmiter RD (1996) Metallothionein I and II protect against zinc deficiency and zinc toxicity in mice. J Nutr 120:403-409.

King CE, Jacobs I, Dickson TC, Vickers JC (1997) Physical damage to rat cortical axons mimics early Alzheimer's neuronal pathology. NeuroReport 8:1663-1665.

King CE, Canty AJ, Vickers JC (2001) Alterations in neurofilaments associated with reactive brain changes and axonal sprouting following acute physical injury to the rat neocortex. Neuropathol Appl Neurobiol 27:115-126.

Lee MK, Cleveland DW (1996) Neuronal intermediate filaments. Annu Rev Neurosci 19:187-217.

Lee MK, Tuttle JB, Rebhun LI, Cleveland DW, Frankfurter A (1990) The expression and posttranslational modification of a neuron-specific betatubulin isotype during chick embryogenesis. Cell Motil Cytoskeleton 17:118-132.

Palmiter RD (1998) The elusive function of metallothioneins. Proc Natl Acad Sci USA 95:8428-8430.

Palmiter RD, Findley SD, Whitmore TE, Durnam DM (1992) MT-III, a brain specific member of the metallothionein gene family. Proc Natl Acad Sci USA 89:6333-6337.

Penkowa M, Hidalgo J (2000) Metallothionein I+II expression and their role in experimental autoimmune encephalomyelitis. Glia 32:247-263.

Penkowa M, Hidalgo J (2001) Metallothionein treatment reduces proinflammatory cytokines IL- 6 and TNF- $\alpha$ and apoptotic cell death during experimental autoimmune encephalomyelitis (EAE). Exp Neurol 170:1-14

Penkowa M, Carrasco J, Giralt M, Moos T, Hidalgo J (1999a) CNS wound healing is severely depressed in metallothionein I- and II-deficient mice. J Neurosci 19:2535-2545.

Penkowa M, Giralt M, Moos T, Thomsen PS, Hernandez J, Hidalgo J (1999b) Impaired inflammatory response to glial cell death in genetically metallothionein-I- and -II-deficient mice. Exp Neurol 156:149-164.

Penkowa M, Carrasco J, Giralt M, Molinero A, Hernandez J, Campbell IL, Hidalgo J (2000) Altered central nervous system cytokine-growth factor expression profiles and angiogenesis in metallothionein-I+II deficient mice. J Cereb Blood Flow Metab 20:1174-1189.

Penkowa M, Giralt M, Camats J, Hidalgo J (2002) Metallothionein 1+2 protect the CNS during neuroglial degeneration induced by 6-aminonicotinamide. J Comp Neurol 444:174-189.

Quaife CJ, Findley SD, Erickson JC, Froelick GJ, Kelly EJ, Zambrowicz BP, Palmiter RD (1994) Induction of a new metallothionein isoform (MTIV) occurs during differentiation of stratified squamous epithelia. Biochemistry 33:7250-7259.

Romero-Isart N, Jensen LT, Zerbe O, Winge DR, Vasak M (2002) Engineering of metallothionein-3 neuroinhibitory activity into the inactive isoform metallothionein-1. J Biol Chem 277:37023-37028.

Sato M, Bremner I (1993) Oxygen free radicals and metallothionein. Free Radic Biol Med 14:325-337.

Sewell AK, Erickson JC, Palmiter RD, Winge DR (1995) Bioactivity of metallothionein-3 correlates with its novel $\beta$ domain sequence rather than metal binding properties. Biochemistry 34:4740-4747.

Trayhurn P, Duncan JS, Wood AM, Beattie JH (2000) Regulation of metallothionein gene expression and secretion in rat adipocytes differentiated from preadipocytes in primary culture. Horm Metab Res 32:542-547.

Trendelenburg G, Prass K, Priller J, Kapinya K, Polley A, Muselmann C, Ruscher K, Kannbley U, Schmitt AO, Castell S, Wiegand F, Meisel A, Rosenthal A, Dirnagl U (2002) Serial analysis of gene expression identifies metallothionein-II as major neuroprotective gene in mouse focal cerebral ischemia. J Neurosci 22:5879-5888.

Uchida Y, Takio K, Titani K, Ihara Y, Tomonaga M (1991) The growth inhibitory factor that is deficient in the Alzheimer's disease brain is a 68 amino acid metallothionein-like protein. Neuron 7:337-347.

Uchida Y, Gomi F, Masumizu T, Miura Y (2002) Growth inhibitory factor prevents neurite extension and death of cortical neurons caused by high oxygen exposure through hydroxyl radical scavenging. J Biol Chem 277:32353-32359.

Ulfig N, Nickel J, Bohl J (1998) Monoclonal antibodies SMI 311 and SMI 312 as tools to investigate the maturation of nerve cells and axonal patterns in human fetal brain. Cell Tissue Res 291:433-443.

Vasak M, Hasler DW (2000) Metallothioneins: new structural and functional insights. Curr Opin Chem Biol 4:177-183. 